\title{
Wheland Intermediates: An ab Initio Valence Bond Study
}

\author{
Mélodie Hadzic, Benoît Braïda, ${ }^{*}$ and François Volatron* \\ UPMC Univ Paris 06, UMR 7616, Laboratoire de Chimie Théorique, case courrier 137, \\ 4, place Jussieu, F-75252 Paris Cedex 05, France, and CNRS, UMR 7616, Laboratoire \\ de Chimie Théorique, 75252 Paris Cedex 05, France
}

benoit.braida@upmc.fr; volatron@lct.jussieu.fr

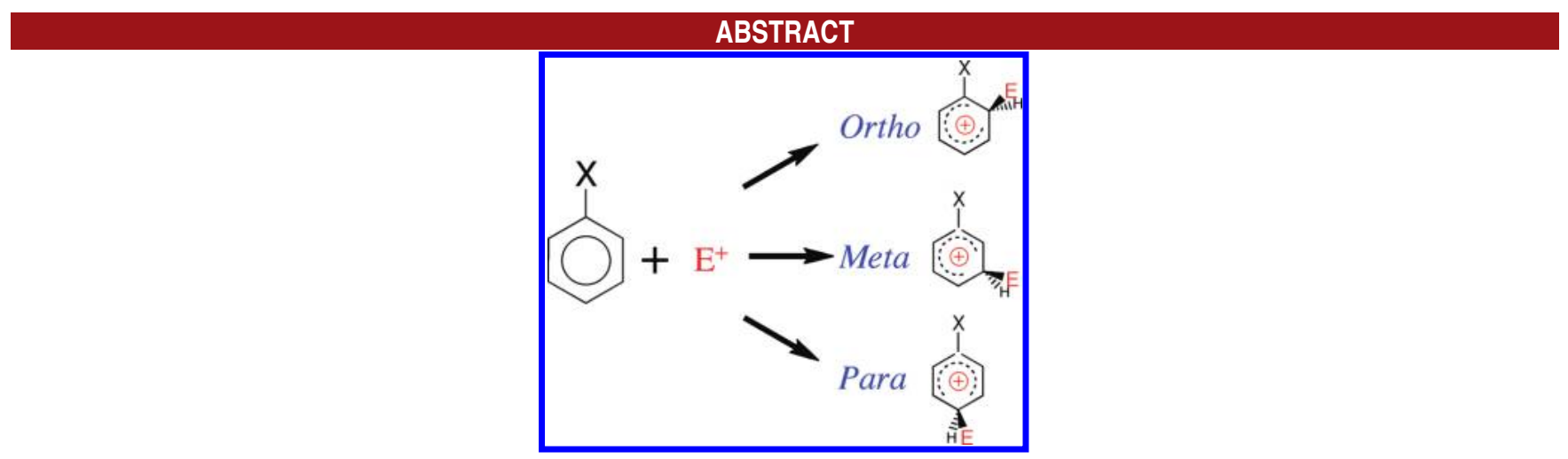

The traditional resonance model for electrophilic attacks on substituted aromatic rings is revisited using high level valence bond (VB) calculations. A large $\pi$-donation is found in the $X=\mathrm{NH}_{2}$ case and a weaker one for the $\mathrm{X}=\mathrm{Cl}$ case, not only for ortho and para isomers but also for the meta case, which can be explained by considering five (not three) fundamental VB structures. No substantial $\pi$-effect is found in the $\mathrm{X}=\mathrm{NO}_{2}$ case, generally viewed as $\pi$-attractive.

The regioselectivity of electrophilic attacks on monosubstituted benzenes is ruled by the electronic nature of the first substituent. Electron donors activate the reaction and direct the second substitution to the ortho and para positions, whereas electron-withdrawing groups are deactivating and meta directing. These observations, also known as Holleman rules, ${ }^{1}$ are usually rationalized through a resonance model analysis. ${ }^{2,3}$ In the case of $\sigma$-attracting and $\pi$-donating groups such as $\mathrm{OH}$ or $\mathrm{NH}_{2}$, the ortho para orientation of the second substitution is rationalized through the analysis of the resonance forms describing the $\pi$-system of the different possible cationic intermediates, also called Wheland intermediates. ${ }^{4}$ From their inspection it is concluded that positions ortho and para for the second substitution are the most favorable in a $\pi$-donating group case, since they allow delocalization of the lone pair into the benzenic moiety, as depicted in

(1) Holleman, A. F. Chem. Rer. 1924, 1, 187.

(2) Smith, M. B.; March, J. March's Advanced Organic Chemistry: Reactions, Mechanisms, and Structure, 6th ed.; Wiley-Intersciences: 2007.

(3) Vollhardt, K. P. C. ; Schore, N. E. Organic Chemistry: Structure and Function, 4th ed.; W. H. Freeman: New York, 2002.

(4) Wheland, G. W. J. Am. Chem. Sod. 1942, 64, 900.
Scheme 1. The VB resonance model is preferred over the simple MO picture in this case, as a HOMO orbital analysis cannot account for the regiospecificities, ${ }^{5 \mathrm{c}}$ and HOMO-LUMO gaps correlate poorly with reactivity. ${ }^{5 f}$

There has been of course many theoretical studies on different aspects of the Electrophilic Aromatic Substitution (EAS), and its precise mechanism is still intensively studied and hotly debated. ${ }^{5}$ In particular, the importance of a cationic $\pi$-intermediate has been studied, ${ }^{5 \mathrm{c}, \mathrm{f}}$ as well as the Single Electron Transfer vs polar (Ingold-Hughes) nature of the mechanism. ${ }^{5 \mathrm{~b}, \mathrm{~d}}$ Models to quantify reactivity from the initial reactant properties has been proposed as well. ${ }^{5 \mathrm{a}, \mathrm{e}}$ However, as the resonance model remains the

(5) See for instance: (a) Vos, A. M.: Nulens, K. H. L.; De Proft, F.; Schoonheydt, R. A.; Geerlings, P. J. Phys. Chem. B 2002, 106, 20262034. (b) Esteves, P. M.; Carneiro, J. W. D.; Cardoso, S. P.; Barbosa, A. G. H.: Laali, K. K.; Rasul, G.; Prakash, G. K. S.; Olah. G. A. I. Am. Chem. Soc. 2003, 125, 4836. (c) Hirao, H.: Ohwada, T. J. Phys. Chem. A 2003, 107, 2875-2881. (d) Lenoir, D. Angew. Chem., Int. Ed. 2003, 42, 854. (e) de Queiroz, J. F.; Carneiro, J. W. D.: Sabino, A. A.; Sparrapan, R.; Eberlin, M. N.; Esteves, P. M. J. Org. Chem. 2006, 71, 6192. (f) Koleva. G.; Galabov, B.; Wu, J. I.; Schaefer, H. F.; Schleyer, P. V. J.Am. Chem. Sod 2009, 131, 14722. (g) Wheeler, S. E.; Houk, K. N. J. Am. Chem. Sod. 2009, 131, 3126. 
Scheme 1. Usual Textbook Resonance Model for Regioselectivity in Electrophilic Aromatic Substitutions (X $=\mathrm{NH}_{2}$ case, ortho and meta isomers)

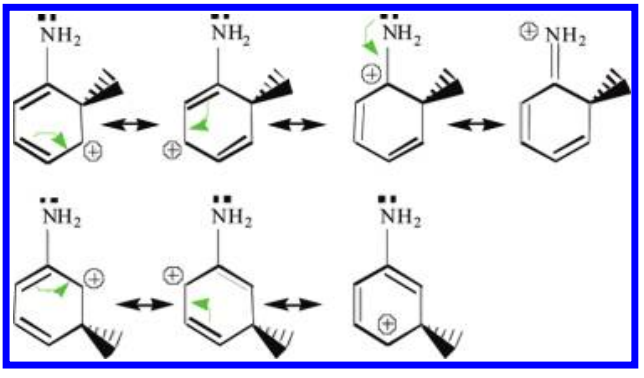

most widely used explanation, and as the resonance concept is attached to Valence Bond (VB) theory, ${ }^{6}$ it is desirable to provide quantitative support for this rationalization of EAS regioselectivity through high level $a b$ initio valence bond (VB) calculations. Quantitative support for this model with a firm theoretical foundation is all the more necessary as the validity of the resonance model has been repeatedly questioned recently, in its common use to explain the rotational barrier of amides ${ }^{7}$ and the acidity of carboxylic acids. ${ }^{8}$ We have thus studied three representative Wheland intermediates with an amino, a chloro, and a nitro group as the first substituent, using the "breathing orbital" valence bond (BOVB) method of Hiberty et al. ${ }^{9}$ Valence Bond calculations have been performed with the XMVB program, ${ }^{10}$ on geometries optimized using Gaussian03 ${ }^{11}$ (see the Supporting Information for a brief description of the BOVB method and computational details). Our selection of molecules thus encompasses three substituents, a strong and a weak $\pi$-donating as well as a $\pi$-attracting substituent, but all three are $\sigma$-attracting. Intermediates under proton attack are considered here for the sake of simplicity. In each case, the three isomers, ortho, meta, and para, are considered.

Let us first consider the Wheland intermediate, i.e. protonated benzene. Three VB structures (I-III) are generally invoked in the description of its $\pi$-electronic system (Scheme 2). ${ }^{3}$ However, structures I and II are the limiting $\pi$-structures of an allylic cation on the $\mathrm{C}_{2} \mathrm{C}_{3} \mathrm{C}_{4}$ moiety, and a structure displaying a "long bond" between

(6) Shaik, S.; Hiberty, P. C. A Chemist's Guide to Valence Bond Theory; WILEY-Interscience: 2007.

(7) (a) Wiberg, K. B.; Laidig, K. E. J. Am. Chem. Soc. 1987, 109, 5935. (b) Wiberg, K. B.; Breneman. C. L. J. Am. Chem. Sod. 1992, 114, 831. (c) Bader, R. F. W. Acc. Chem. Res. 1985, 18,9.

(8) (a) Siggel, M. R.; Thomas, T. D. J. Am. Chem. Sod. 1986, 108. 4360. (b) Siggel, M. R. F.; Streitwieser, A, Jr.; Thomas, R. D. J. Am. Chem. Sod. 1988, 110, 8022

(9) (a) Hiberty, P. C.; Flament, J. P.; Noizet, E. Chem. Phys. Lett. 1992, 189, 259. (b) Hiberty, P. C.; Humbel, S.; Byrman, C. P.; van Lenthe, J. H. J. Chem. Phys. 1994, 101, 5969. (c) Hiberty, P. C.; Shaik, S. Theor. Chem. Acc. 2002, 108, 255.

(10) (a) Song, L. ; Mo, Y.; Zhang, Q. ; Wu, W. XMVB: An Ab Initio Non orthogonal Valence Bond Program: Xiamen University, Xiamen, 1999. (b) Song, L.; Mo, Y.; Zhang, Q.; Wu, W. J. Comput. Chem. 2005, 26, 514. (c) Song, L.; Song, J.; Mo, Y.; Wu, W. J. Comput. Chem. 2009, 30, 399.

(11) Frisch, M. J. et al. Gaussian 03, revision D.01; Gaussian Inc.: Wallingford, CT, 2004.
Scheme 2. Five-Structure VB Description of Protonated Benzene

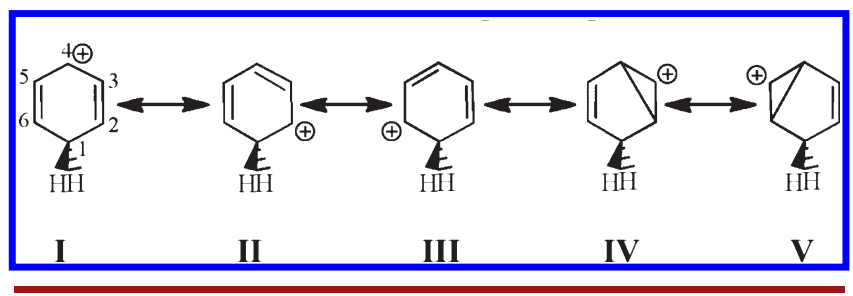

$\mathrm{C}_{2}$ and $\mathrm{C}_{4}$ (structure $\mathbf{I V}$ ) must be taken into account to obtain a correct description of the $\pi$-system, as has been first proposed by Harcourt for three-center four-electrons $\pi$-systems, ${ }^{12}$ and later confirmed on an allyl cation and anion. ${ }^{13,14}$ Similarly, a description of the allylic cation situation on the $\mathrm{C}_{4} \mathrm{C}_{5} \mathrm{C}_{6}$ moiety needs a long-bonded structure $\mathbf{V}$ in addition to I and III. Our BOVB calculations confirm the importance of these two extra structures IV and $\mathbf{V}$ with a $14.5 \%$ computed weight, not so far from the $20.2 \%$ weight found for structures II and III.

Table 1. Relative Energies (in $\mathrm{kcal} / \mathrm{mol}$ ) of the Different Wheland Intermediates at the BOVB (CCSD(T)) Levels

\begin{tabular}{lccc}
\hline$-\mathrm{X}$ & $-\mathrm{NH}_{2}$ & $-\mathrm{Cl}$ & $-\mathrm{NO}_{2}$ \\
\hline Ortho & $10.7(4.6)$ & $3.8(1.7)$ & $0.6(0.3)$ \\
Meta & $30.4(28.4)$ & $7.8(7.6)$ & $0.0(0.0)$ \\
Para & $0.0(0.0)$ & $0.0(0.0)$ & $4.2(1.5)$
\end{tabular}

At least five structures will hence be used in the following. All structures, including structures coming from the development of each covalent $\pi$-bond into its corresponding ionic components, will be neglected as they are not expected to significantly change the relative $\pi$-effect in the different intermediates. This hypothesis is checked by comparing the relative energies of the different Wheland intermediates, at the BOVB and $\mathrm{CCSD}(\mathrm{T})$ levels respectively (Table 1). ${ }^{15}$ BOVB calculations are in correct agreement with those obtained at the $\operatorname{CCSD}(\mathrm{T})$ level, leading to an energy ranking of the different isomers that is accurate enough for the purpose of this study.

(12) Harcourt, R. D.; Roso, W. Can. J. Chem. 1978, 56, 1093

(13) (a) Mo, Y.; Lin, Z.; Wu, W.; Zhang, Q. J. Phys. Chem. 1996, 100, 6469. (b) Linares, M.; Humbel, S.; Braida, B. J. Phys. Chem. A 2008, 112, 13249.

(14) Actually, this "long bonded" structure for an allyl cation can be retrieved from simple MO analysis. The lowest molecular orbital $\pi_{1}$ in simple Hückel theory is $\pi_{1}=1 / 2\left(p_{1}+\sqrt{2} p_{2}+p_{3}\right)$. The ground state of this cation may be then developed on the atomic orbitals basis (discarding the normalization factor):

$$
\begin{aligned}
\left|\pi_{1} \overline{\pi_{1}}\right|= & \left(\left|p_{1} \overline{p_{1}}\right|+2\left|p_{2} \overline{p_{2}}\right|+\left|p_{3} \overline{p_{3}}\right|\right)+\sqrt{2}\left(\left|p_{1} \overline{p_{2}}\right|+\left|p_{2} \overline{p_{1}}\right|\right) \\
& +\sqrt{2}\left(\left|p_{3} \overline{p_{2}}\right|+\left|p_{2} \overline{p_{3}}\right|\right)+\left(\left|p_{1} \overline{p_{3}}\right|+\left|p_{3} \overline{p_{1}}\right|\right)
\end{aligned}
$$

The three first terms account for ionic structures, and then one can find the classical VB scheme of a covalent bond between $C_{1}$ and $C_{2}$ and $C_{2}$ and $\mathrm{C}_{3}$ respectively. The last term does not correspond to a classical $\mathrm{VB}$ structure, and it describes a long bond between $\mathrm{C}_{1}$ and $\mathrm{C}_{3}$.

(15) B3LYP results (not shown here) give very similar energy differences as $\operatorname{CCSD}(\mathrm{T})$ values. 
For all substituted arenium ions, two BOVB wave functions have been computed: the first one including the five fundamental structures $(\mathbf{I}-\mathbf{V})$, and the second one that includes extra structures describing the substituent $\pi$-effect. The energy differences between the two BOVB wave functions (eq 1) gives the energetic stabilization due to the substituent $\pi$-effect (Table 2).

$$
E_{\pi \text {-effect }}=E\left(\Psi_{\mathrm{I}-\mathrm{V}+\pi}^{\mathrm{BOVB}}\right)-E\left(\Psi_{\mathrm{I}-\mathrm{V}}^{\mathrm{BOVB}}\right)
$$

As the $\pi$-effect is expected to be switched off when $-\mathrm{NH}_{2}$ or $-\mathrm{NO}_{2}$ groups are perpendicular to the benzene plane, we expect the rotation barrier around the $\mathrm{C}-\mathrm{N}$ bond to provide similar trends for the various isomers as VB computed $\pi$-delocalization energies. We have thus computed the rigid rotation barrier at the CCSD(T) level, by a $90^{\circ}$ rotation of the substituent. These calculations will only provide an approximate evaluation of the $\pi$-effect, because other effects are affected when the substituents are rotated (electrostatic effects, hyperconjugation effects, ...). Results are also displayed in Table 2 (in parentheses). We see that a very similar trend is obtained, whether the true $\pi$-effect computed at the VB level is considered or its approximate evaluation through the rigid rotation barrier.

Table 2. $\pi$-Effect (in $\mathrm{kcal} / \mathrm{mol}$ ) Stabilization for Each Wheland Intermediate, As Defined by eq 1, and in Parentheses Rigid Rotation Barriers Computed at the CCSD(T) Level

\begin{tabular}{llrr}
\hline$-\mathrm{X}$ & $-\mathrm{NH}_{2}$ & $-\mathrm{Cl}$ & $-\mathrm{NO}_{2}$ \\
\hline Ortho & $-36.4(-37.7)$ & -6.9 & $-0.8(-3.8)$ \\
Meta & $-17.0(-12.6)$ & -3.8 & $-1.7(-6.6)$ \\
Para & $-43.1(-41.7)$ & -11.4 & $0.0(-2.1)$ \\
\hline
\end{tabular}

Let us first consider the case where $\mathrm{X}=\mathrm{NH}_{2}$ which is a strong $\pi$-donor. The different structures, along with the computed weights, are displayed in Figure 1. The $\pi$-donation ability of this substituent is characterized by the existence of an additional mesomeric structure VI in the ortho and para isomers. However, such an extra $\pi$-donating structure can be generated as well for the meta isomer, from the "long bonded" structure IV. For ortho and para positions this extra structure is generated from a dominant structure (II and I respectively), whereas in the meta isomer it arises from a presumably less important structure (IV). Our VB calculations give a very large weight (more than $32 \%$ ) for structure VI in both ortho and para intermediates and a much smaller $(14.5 \%)$ one for the meta isomer. If one considers the summed weight of the two structures that together describes a carbon-nitrogen $\pi$-bond, i.e. II $+\mathbf{V I}$ and $\mathbf{I}+\mathbf{V I}$ for ortho and para cases respectively, it indeed amounts to almost $\sim 70 \%$, leaving the benzene ring resonance as only a secondary stabilizing component for these compounds. The weight of structure VI is still significant in the meta isomer, indicating that the amino group appears to have a significant $\pi$-donating effect in the meta isomer. The $\pi$-energy stabilizations displayed in Table 2 lead to the same conclusions, as large energetic $\pi$-effects are measured for all three isomers, including the meta one, although for this latter it is more than twice smaller than that for the other isomers. These large stabilizations are compatible with the amino substituent as being an "activator". The large $\pi$-donation dominates over the $\sigma$-attracting effect, finally leading to a lower barrier under an electrophilic attack than for the nonsubstituted case.

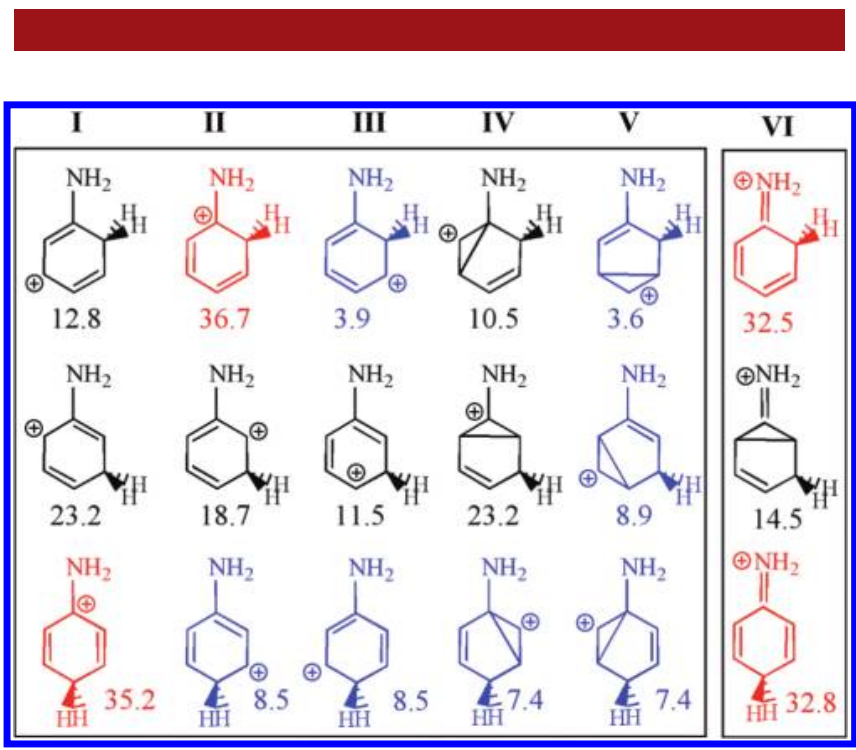

Figure 1. Computed BOVB weights (in \%), for the ortho, para, and meta isomers of $\mathrm{C}_{6} \mathrm{H}_{6} \mathrm{NH}_{2}{ }^{+}$.

Let us switch to the case where $\mathrm{X}=\mathrm{Cl}$. As the chloro substituent is $\pi$-donating, the $\mathrm{VB}$ description is exactly the same as for the $\mathrm{X}=\mathrm{NH}_{2}$ case (Figure 2). The lower values for the weights of structure VI mainly highlight the lower $\pi$-effect of the chlorine as compared to the amino substituent. The $\pi$-effect energy stabilizations (Table 2) confirm these observations, revealing the same ordering of the substituent $\pi$-effect, but with values considerably reduced as compared to the $\mathrm{X}=\mathrm{NH}_{2}$ case.

This much weaker $\pi$-effect for chlorine may not be sufficient to counterbalance a stronger $\sigma$-attracting (destabilizing) effect as compared with the amino group, thus leading the chlorine to act as a "deactivator". However, the chlorine mesomeric effect is at the same time significant enough to be at the origin of the para (and less importantly ortho) preferential addition observed for $\mathrm{C}_{6} \mathrm{H}_{6} \mathrm{Cl}$.

Last, the nitro group, considered as a strong electronwithdrawing substituent, ${ }^{16}$ is considered with nitrobenzene. Delocalization of a benzene electron pair on the nitro group generates structures with formally two positive charges on the benzene ring, as shown in Figure 3. We have selected only the structures that do not display two neighboring positive charges, which leads to two (three) extra structures for the ortho (meta) isomers and none for

(16) (a) Hansch, C.; Leo, A.; Taft, R. W. Chem. Rer. 1991, 91, 165. (b) Feuer, H. In The Chemistry of Nitro and Nitroso Groups; Patai, S., Ed.; WILEY: New York, 1969. 

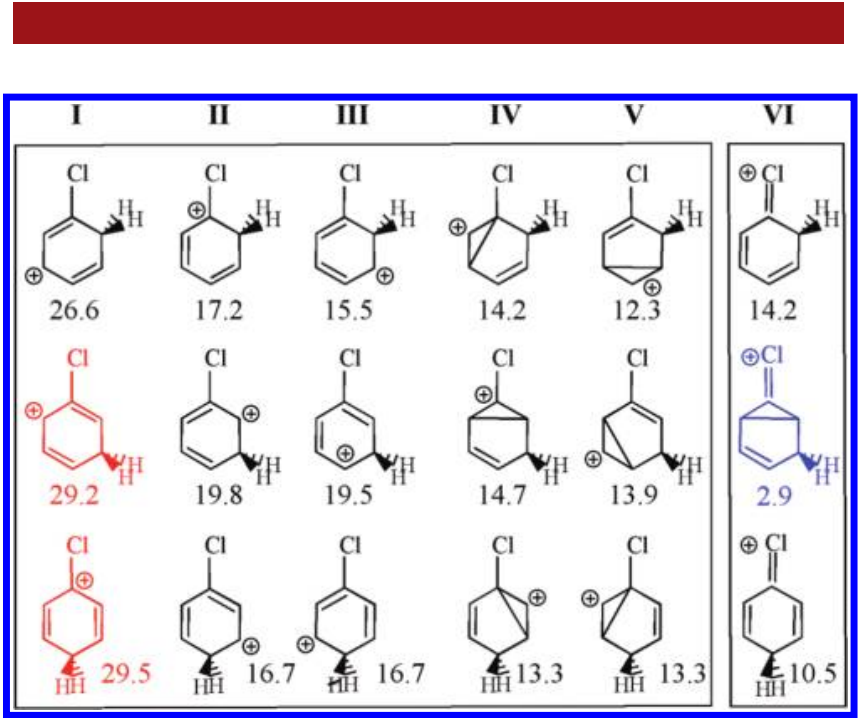

Figure 2. Computed BOVB weights (in \%), for the ortho, para, and meta isomers of $\mathrm{C}_{6} \mathrm{H}_{6} \mathrm{Cl}^{+}$.

the para one. These extra structures do not contribute much to the mesomery, since the structure weights are small, and so is the $\pi$-energy stabilization. In all isomers a structure with a positive charge on the carbon vicinal to the nitro group is found (II, IV, and I in ortho, meta, and para isomers respectively) the weight of which is significant, between 11 and 26\%, although these structures are generally considered to be negligible. The three isomers have almost the same energy (Table 1) within $2 \mathrm{kcal} / \mathrm{mol}$, a result in accordance with the observed products of nitration of nitrobenzene, where a mixture of ortho $(6 \%)$, meta $(93 \%)$, and para $(1 \%)$ isomers is experimentally obtained. ${ }^{2}$

All in all, our high level ab initio Valence Bond calculations provide quantitative support for the traditional resonance model for the second electrophilic attack on substituted benzenes. However, they also reveal that the usual textbook presentation should be complemented and some elements modified. For an amino substituent, the ortho/para preferential attack has been traced back to a stronger $\pi$-donating stabilization than in the meta case, the $\pi$-effect being significant in the latter case though. A

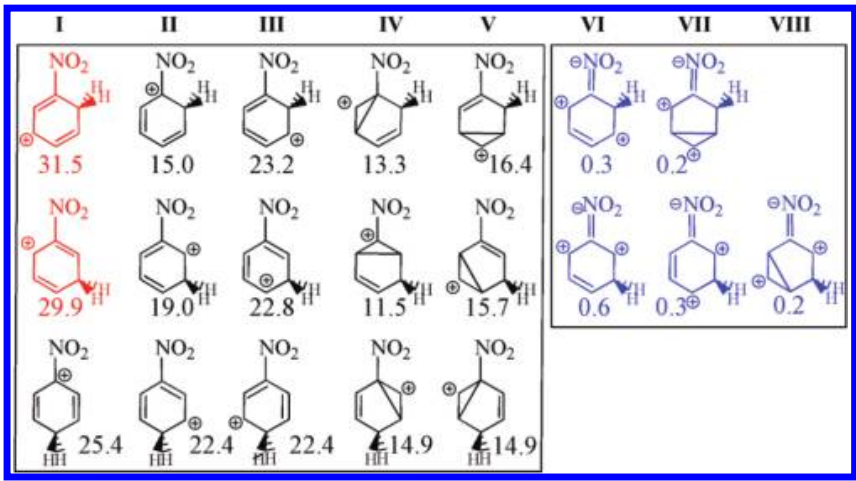

Figure 3. Computed BOVB weights (in \%), for the ortho, para, and meta isomers of $\mathrm{C}_{6} \mathrm{H}_{6} \mathrm{NO}_{2}{ }^{+}$.

strong $\pi$-effect in the meta isomer can only be understood if the resonance model includes five fundamental resonance contributors, and not only three as in the usual oversimplified model. The strong substituent $\pi$-stabilization is most likely to be the key factor explaining its "activator" behavior, offsetting the $\sigma$-attracting (destabilizing) effect. In the nitro case, the three isomers appear to lie close in energy, and the weights of the extra " $\pi$-effect" structures, as well as the corresponding energy stabilization, are very small. Hence, in opposition to what can be thought, the nitro group does not show any significant $\pi$-attracting effect in Wheland intermediates. In particular, the fundamental structure with a positive charge on the carbon bearing the nitro group does not appear to be a minor contributor, contrary to the explanation sometimes given in this case. ${ }^{3}$ The "deactivator" behavior of the $\mathrm{NO}_{2}$ substituent is thus more likely to be related to a $\sigma$-attracting (destabilizing) effect which is not counterbalanced by a $\pi$-stabilizing effect. 